\title{
Electric field tuning of hard polar phonons in strained $\mathrm{SrTiO}_{3}$ films
}

V. Skoromets, C. Kadlec, P. Kužel, S. Kamba, and J. Schubert

Citation: Journal of Applied Physics 107, 124116 (2010);

View online: https://doi.org/10.1063/1.3447812

View Table of Contents: http://aip.scitation.org/toc/jap/107/12

Published by the American Institute of Physics

\section{Articles you may be interested in}

Field-induced soft mode hardening in $\mathrm{SrTiO}_{3} / \mathrm{DyScO}_{3}$ multilayers

Applied Physics Letters 93, 052910 (2008); 10.1063/1.2967336

The low-temperature infrared optical functions of $\mathrm{SrTiO}_{3}$ determined by reflectance spectroscopy and spectroscopic ellipsometry

Journal of Applied Physics 78, 1235 (1998); 10.1063/1.360364

Dielectric tunability of $\mathrm{SrTiO}_{3}$ thin films in the terahertz range

Applied Physics Letters 88, 102901 (2006); 10.1063/1.2183370

Electrically tunable near-field radiative heat transfer via ferroelectric materials

Applied Physics Letters 105, 244102 (2014); 10.1063/1.4904456

Mid-infrared time-domain ellipsometry: Application to Nb-doped $\mathrm{SrTiO}_{3}$

Applied Physics Letters 101, 081103 (2012); 10.1063/1.4746263

Soft mode behavior in $\mathrm{SrTiO}_{3} / \mathrm{DyScO}_{3}$ thin films: Evidence of ferroelectric and antiferrodistortive phase transitions

Applied Physics Letters 95, 232902 (2009); 10.1063/1.3271179

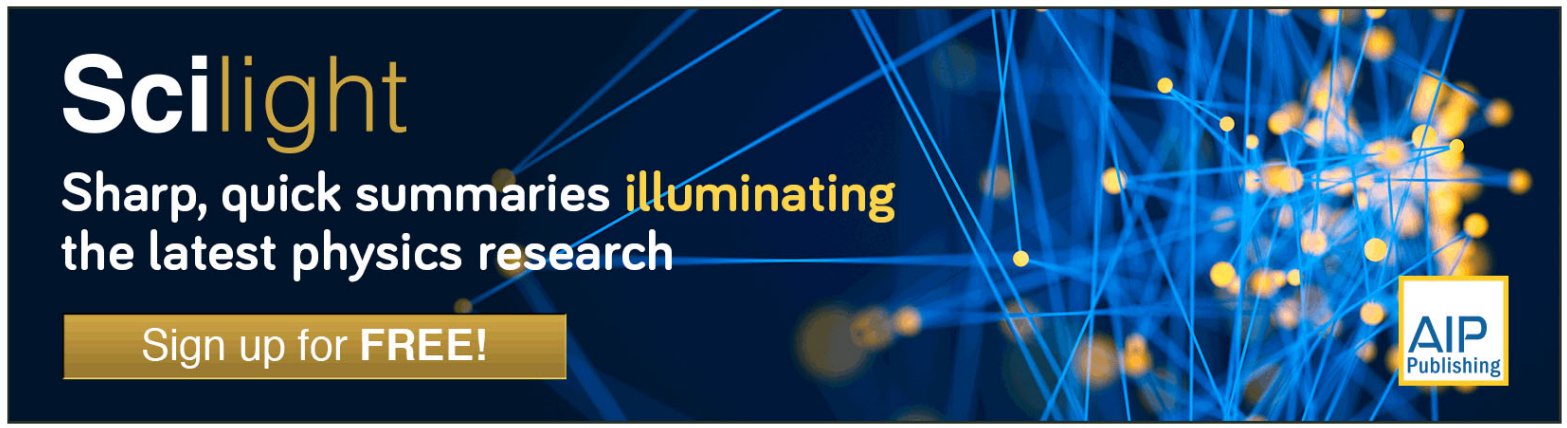




\title{
Electric field tuning of hard polar phonons in strained $\mathrm{SrTiO}_{3}$ films
}

\author{
V. Skoromets, ${ }^{1}$ C. Kadlec, ${ }^{1}$ P. Kužel, ${ }^{1, a)}$ S. Kamba, ${ }^{1}$ and J. Schubert ${ }^{2}$ \\ ${ }^{1}$ Institute of Physics, Academy of Sciences of the Czech Republic, Na Slovance 2, 18221 Prague 8, \\ Czech Republic \\ ${ }^{2}$ Institute of Bio- and Nanosystems, IBN-1, Forschungszentrum Jülich GmbH and Jülich-Aachen Research \\ Alliance, JARA-FIT, Jülich 52425, Germany
}

(Received 7 May 2010; accepted 10 May 2010; published online 28 June 2010)

\begin{abstract}
We investigate $100 \mathrm{~nm}$ thick epitaxial monolayer of $\mathrm{SrTiO}_{3}$ strained by $1 \%$ in biaxial tension and grown on (110) $\mathrm{DyScO}_{3}$. Spectra within the whole far-infrared spectral range are obtained as a function of temperature and an electric bias. An appreciable tunability of high frequency polar phonons is observed upon bias. The splitting of TO4 mode clearly observed below $220 \mathrm{~K}$, which is further increased upon bias, is a signature of the ferroelectricity of the film at these temperatures.
\end{abstract}

(C) 2010 American Institute of Physics. [doi:10.1063/1.3447812]

\section{INTRODUCTION}

Investigation of tunable dielectric properties of materials is important for the development of components enabling the control of the propagation of radiation in the subterahertz and terahertz region. Ferroelectric materials are characterized by a high permittivity up to the far-infrared range (FIR). This is due to the polar soft mode and/or polar relaxations connected to the ferroelectric instability. Usually, these materials may be tuned by means of temperature or external low frequency electric field. ${ }^{1}$

Strontium titanate $\left(\mathrm{SrTiO}_{3}, \mathrm{STO}\right)$ is an incipient ferroelectric and the dielectric behavior of STO single crystals is determined by the soft mode which exhibits a frequency decrease upon cooling. This leads to an increase in its permittivity and of its electric field tunability with decreasing temperature. The electric field tuning of the ferroelectric soft mode in bulk single crystals at low temperatures was first reported by Schaufele et al. ${ }^{10}$ and by Worlock and Fleury. ${ }^{11}$ An appreciable hardening of the soft mode along with a small hardening of TO2 mode was observed also in thin films. ${ }^{12}$ These properties along with the well developed technology for strained or unstrained STO thin film deposition make this material highly promising for applications in the microwave components.

Indeed, one of the important factors which affect the ferroelectric properties of STO is the strain. Strained films can be achieved by STO deposition on substrates with a slightly different lattice constant from that of STO. By selecting the lattice parameter mismatch between STO and the substrate material one controls the sign and magnitude of the in-plane strain in the thin films. Ferroelectric phase transition can be induced by a tensile strain ${ }^{2,3}$ and the transition temperature can be increased up to the room temperature by the choice of a suitable substrate.

$\mathrm{DyScO}_{3}$ (DSO) has been extensively used as an appropriate substrate for highly strained STO films. ${ }^{2-9}$ The investigation of the material tunability has been carried out in the gigahertz and terahertz spectral ranges ${ }^{2,4-8}$ and the observed

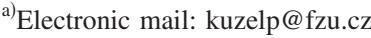

dielectric behavior has been described by the ferroelectric soft mode and a coupled dielectric relaxation. In this paper we report on an electric field-induced tuning of higher frequency (hard) modes in a $100 \mathrm{~nm}$ thick single layer of STO deposited on DSO substrate.

\section{EXPERIMENTAL}

The $100 \mathrm{~nm}$ thick STO film was prepared by pulsed laser deposition on (110)-oriented $10 \times 10 \times 0.9 \mathrm{~mm}^{3}$ DSO substrate in an on-axis geometry. A variable dc electric bias was applied by using interdigitated electrode structure deposited on top of the film (see Ref. 5 for more technological details). The mode tuning was investigated by Fourier spectrometer Bruker IFS113v equipped with a helium-cooled Si bolometer. It was adjusted for reflectance measurements; the transmission mode could not be used due to the absorption of far-infrared radiation on phonon modes of the DSO substrate.

In a usual experimental setup the input face of the sample is in contact with a metallic aperture which defines the probed area of the sample. This was not possible to achieve in our case owing to the presence of the conductive electrode structure and electrical contacts on top of the film. The probing infrared radiation thus illuminated not only the active area of the sample (part covered with the electrodes) but also other parts of the sample in our experiments. Schematic sample layout is shown in Fig. 1. Linearly polarized

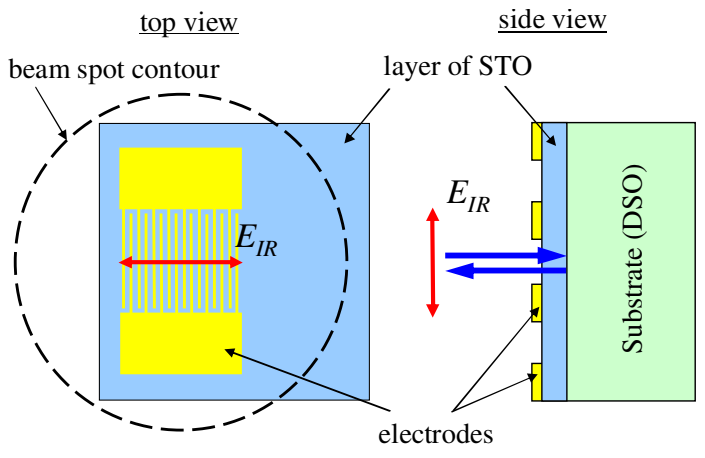

FIG. 1. (Color online) Sample layout. 


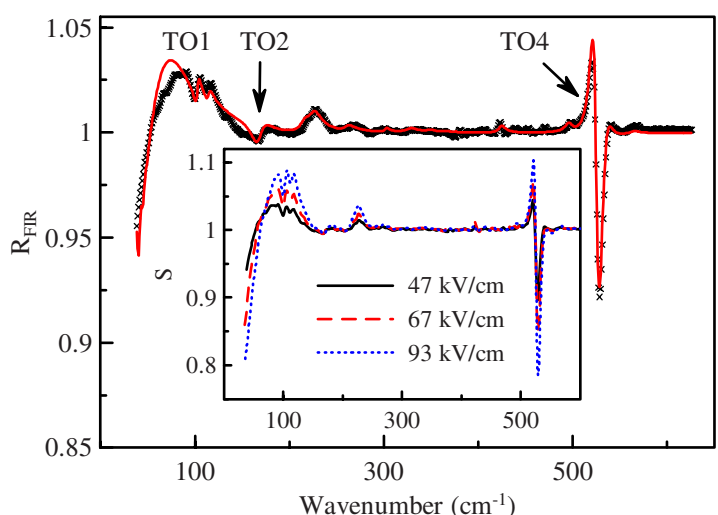

FIG. 2. (Color online) Comparison of normalized experimental (symbols) reflectance ratio $R_{\mathrm{FIR}}$ with its fit (solid line); room temperature, $47 \mathrm{kV} / \mathrm{cm}$ bias. Inset: raw reflectance ratio $S$ at room temperature under three different bias fields.

radiation was used in experiments with the electric field vector perpendicular to the electrode fingers and parallel to [110] direction of DSO. In this geometry the electrode structure was transparent for the FIR radiation and had the smallest influence on the reflected signal. For each measurement a reference spectrum $R_{0}(\omega)$ was first measured without an electric field; subsequently a dc field $E$ was applied and a reflectance spectrum $R_{E}(\omega)$ was obtained. The ratio $S(\omega)$ of these two experimental spectra reads

$$
S=\frac{R_{E}}{R_{0}}=\frac{I_{E}+I_{S}}{I_{0}+I_{S}}
$$

where $I_{E}$ is the infrared power reflected from the active area of the sample with the bias field on, $I_{0}$ is the power coming from the active area with the bias field off, and $I_{S}$ is a signal reflected from outside the active area. The net relative change in the FIR reflectance due to the bias (field-induced reflectance, i.e., $\left.I_{E} / I_{0}=R_{\mathrm{FIR}}\right)$ then reads

$$
R_{F I R}=(S-1) A+1,
$$

where $A=1+I_{S} / I_{0}$. The term $S$ is the raw experimental signal in Eq. (1). We show in the inset of Fig. 2 such raw reflectance curves measured at room temperature and at three different electric fields. The factor $A$ describes the deviation due to the larger beam spot. We tried to estimate this deviation from the low frequency behavior (below $100 \mathrm{~cm}^{-1}$ ) of the spectra. In order to obtain semiquantitative results we assumed that $A$ is a frequency independent parameter. This is a reasonable first-order approximation since it is primarily related to the ratio of the surfaces of the active and inactive area. The low frequency part of the spectra is known from terahertz time-domain experiments ${ }^{7,8}$ and it is governed by the ferroelectric soft mode $\left(\sim 50 \mathrm{~cm}^{-1}\right)$ coupled to a relaxation $\left(\sim 10 \mathrm{~cm}^{-1}\right)$. The expected field-induced reflectivity spectrum $R_{\mathrm{THz}}$ in the frequency range $50-100 \mathrm{~cm}^{-1}$ can be evaluated from the terahertz data. The parameter $A$ was then chosen such that the closest match between $R_{\mathrm{FIR}}$ and $R_{\mathrm{THz}}$ is obtained.

\section{RESULTS AND DISCUSSION}

An example of experimental normalized signal $R_{\mathrm{FIR}}$ is shown in Fig. 2. The substrate has centrosymmetric structure and does not show any electric field tunability. ${ }^{6}$ Obviously, any value in the obtained spectra which differs from unity is caused by electric field-induced changes in the dielectric properties of the thin film which modify the absorption of radiation in the film and the impedance mismatch at air/STO and STO/DSO interfaces. The last phenomenon is at the origin of the fact that a phonon structure of DSO may appear to some extent in the measured field-induced reflectance spectra. This is the case, e.g., for the peak near $225 \mathrm{~cm}^{-1}$ (see Fig. 2) where the tails of the tunable modes of STO overlap with a strong phonon of DSO showing a reststrahlen band below $220 \mathrm{~cm}^{-1}$.

Two prominent features are observed in the experimental spectra (Fig. 2). These are the ferroelectric soft mode (TO1) and TO4 mode. A small signature of the TO2 mode tuning is also observed as discussed later. The soft mode was modeled as a damped oscillator with a bare frequency $\omega_{0}$, damping $\Gamma$, and oscillator strength $f$. It was coupled (coupling constant $\delta)$ to a low frequency relaxation with damping $\gamma$ and strength g. ${ }^{7}$ Hard modes were introduced in the model as independent underdamped harmonic oscillators

$$
\begin{aligned}
\varepsilon(\omega)= & \frac{f(1-i \omega / \gamma)+g\left(\omega_{0}^{2}-\omega^{2}-i \omega \Gamma\right)+2 \delta \sqrt{f g}}{\left(\omega_{0}^{2}-\omega^{2}-i \omega \Gamma\right)(1-i \omega / \gamma)-\delta^{2}} \\
& +\sum_{i=2,4} \frac{f_{\mathrm{TO} i}}{\omega_{\mathrm{TO} i}^{2}-\omega^{2}-i \omega \Gamma_{\mathrm{TO} i}}+\varepsilon_{\infty} .
\end{aligned}
$$

We calculated the reflectance of the structure using the transfer matrix formalism. ${ }^{13}$ Equation (3) was used to model the permittivity of STO and the substrate properties were calculated by using a four parameters model ${ }^{14}$ for the permittivity of DSO (the reflectance spectra of bare DSO were measured and fitted independently in our laboratory and are not shown here).

An example of the reflectance fit is shown in Fig. 2. The parameters describing the permittivity of DSO were not allowed to change during fitting. All the parameters which are involved in the first term of (3) were taken from Refs. 7 and 8 and were also kept constant during fitting; the second term of (3) contains the fitted parameters. The oscillator strength $f_{\mathrm{TO} 4}$ and the damping $\Gamma_{\mathrm{TO} 4}$ were assumed independent of the electric field and temperature. The TO4 mode eigenfrequency was assumed to depend both on temperature and electric bias such as: $\omega_{\mathrm{TO} 4}(T, E)$. Note that for a given temperature $T$ each spectrum (measured with an applied field $E$ ) depends on both $\omega_{\mathrm{TO} 4}(T, E)$ and $\omega_{\mathrm{TO} 4}(T, 0)$, where $\omega_{\mathrm{TO} 4}(T, 0)$ keeps the same value within such series.

TO2 mode of STO appears in the strained film near $169 \mathrm{~cm}^{-1}$ as a tiny dip in the spectra (Fig. 2). Although the electric field tunability of its eigenfrequency $\left(\sim 1 \mathrm{~cm}^{-1}\right.$ at 67 $\mathrm{kV} / \mathrm{cm}$ and room temperature) is comparable to that of TO4 mode, the sensitivity of $R_{\mathrm{FIR}}$ is dramatically different for these two modes. Again this is related to the impedance mismatch between STO and DSO such as: we found a large contrast of refractive indices in the spectral range of TO4 


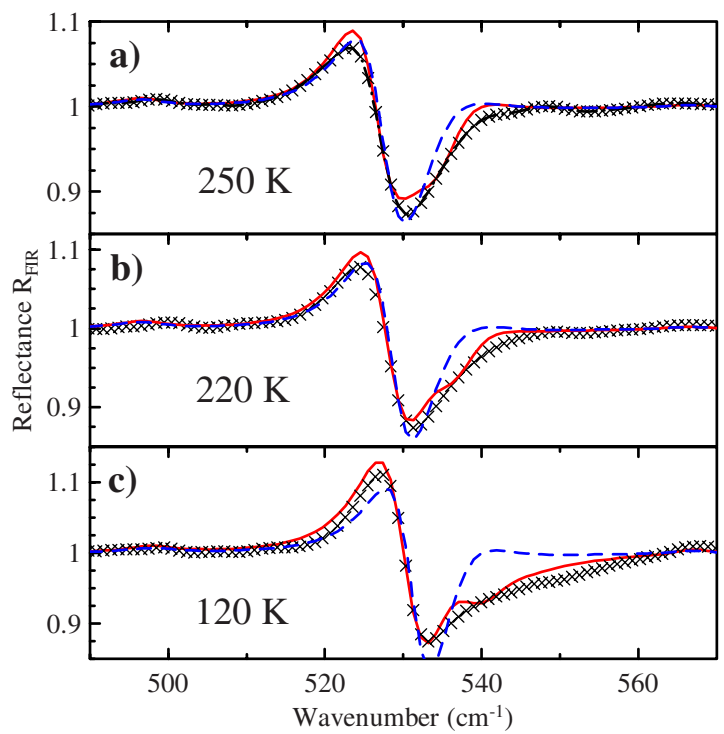

FIG. 3. (Color online) Comparison of high frequency part of experimental (symbols) and fitted (lines) reflectance $\mathrm{R}_{\mathrm{FIR}}$ for the bias field of $67 \mathrm{kV} / \mathrm{cm}$. Solid line includes TO4' mode, dashed line does not.

mode $\left(n_{\mathrm{STO}} \sim 3.8, n_{\mathrm{DSO}} \sim 0.3\right)$ while this contrast is small close to TO2 mode $\left(n_{\mathrm{STO}} \sim 5.5, n_{\mathrm{DSO}} \sim 6.5\right)$. For this reason we obtain only a rough estimation of TO2 mode tuning and we are not able to present any deeper analysis here. Akimov et al. $^{12}$ in their work reported a similar hardening of TO2 mode. The silent TO3 mode has not been detected in our experiments, however, it was observed under applied electric field by Raman scattering both in bulk STO (Ref. 11) and in a thin film sample. ${ }^{12}$

Signatures of TO4 mode splitting are observed in some spectra (Fig. 3). In connection with this phenomenon we supplemented the model with an additional harmonic oscillator which describes the new mode denoted TO4'. Here again we assumed the dielectric strength $f_{\mathrm{TO}}$ ' and the damping $\Gamma_{\mathrm{TO} 4}$, field and temperature independent, and the bare frequency $\omega_{\mathrm{TO} 4}$, field and temperature dependent. The splitting is clearly observed at $220 \mathrm{~K}$ and it becomes highly pronounced at lower temperatures. The behavior at $250 \mathrm{~K}$ cannot be unambiguously determined [Fig. 3(a)]; however, we can state that the fit including $\mathrm{TO}^{\prime}$ mode yields a twice reduced least square sum. These findings are in qualitative agreement with the splitting of TO4 mode observed on a similar film below $200 \mathrm{~K}$ in reflectance spectra polarized along [001]. ${ }^{9}$ In our work the splitting is observed well above the reported antiferrodistortive phase transition occurring (at $\sim 180 \mathrm{~K}),{ }^{9}$ we thus conclude that it is related to the symmetry breaking at the ferroelectric phase transition which occurs slightly above $250 \mathrm{~K}$ in similar films. ${ }^{8}$

In Fig. 4 we present the tuning of the TO4 mode frequencies at various temperatures as supplied by the fits. One can conclude that the tuning shows linear dependence on the bias field. The field-induced hardening of $\omega_{\mathrm{TO}}$ is of up to $2 \mathrm{~cm}^{-1}$ for the maximum bias, while the split mode TO4' hardens by $8 \mathrm{~cm}^{-1}$ at the lowest $(120 \mathrm{~K})$ temperature. In the inset of Fig. 4 the splitting of TO4 mode is shown below 220 $\mathrm{K}$ for zero and maximum $(93 \mathrm{kV} / \mathrm{cm})$ bias. It is seen that the bias field enhances the splitting of the modes. The tiny fre-

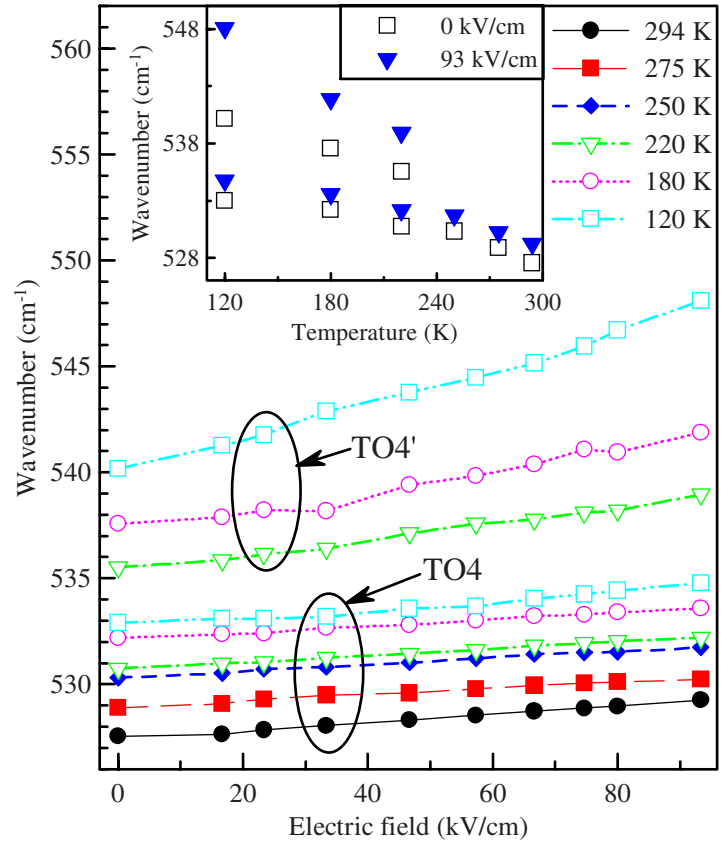

FIG. 4. (Color online) Tuning of the TO4 hard mode by the electric field at different temperatures. Empty symbols belong to split modes. Inset: temperature dependence of the hard modes frequency.

quency changes in the modes $(0.4 \%$ and $1.5 \%$ for $\mathrm{TO} 4$ and TO4', respectively) with respect to their absolute frequency value can be easily detected due to an underdamped nature $\left(\Gamma_{\mathrm{TO} 4} \approx 13 \mathrm{~cm}^{-1}\right)$ of the high frequency modes in STO film. Within unbiased film we observe the splitting of modes of about $7 \mathrm{~cm}^{-1}$ with [110] polarization of far-infrared radiation while a splitting of $\sim 14 \mathrm{~cm}^{-1}$ was reported in Ref. 9 with [001] polarization. This might be a signature of the lack of fourfold axis in the strained sample.

Eigenvectors of polar phonons in $\mathrm{SrTiO}_{3}$ are mixed, therefore, the change in soft mode frequency should influence other polar modes. This statement follows also from the first principle calculations of perovskite oxides, which shows that the longitudinal phonon frequency of the TO1 soft mode corresponds to the mode usually denoted as LO4 mode near $800 \mathrm{~cm}^{-1}$.

\section{CONCLUSION}

We have demonstrated the phenomenon of the electric field-induced tuning of high frequency polar phonons in the strained $100 \mathrm{~nm}$ thick film of STO. The tuning of TO1 soft mode, mainly responsible for the tunability of the low frequency permittivity, was discussed in details previously in Refs. 5-8, 11, and 12. Here we demonstrate a comparatively smaller tuning of hard TO2 and TO4 modes. We focused our quantitative analysis on the field-induced hardening of TO4 mode and on the splitting of this mode below $220 \mathrm{~K}$ due to the ferroelectric phase transition. This splitting exhibits dependence on the applied bias field.

\section{ACKNOWLEDGMENTS}

This work was supported by the Academy of Sciences of the Czech Republic (Project No. AVOZ10100520), by its 
Grant Agency (Project No. A100100907), and by the Czech Science Foundation (Project No. 202/09/0682).

${ }^{1}$ A. Eriksson, A. Deleniv, and S. Gevorgian, J. Appl. Phys. 93, 2848 (2003); J. Dec, W. Kleemann, and B. Westwanski, J. Phys.: Condens. Matter 11, L379 (1999); H.-M. Christen, J. Mannhart, E. J. Williams, and C. Gerber, Phys. Rev. B 49, 12095 (1994); D. Fuchs, C. W. Schneider, R. Schneider, and H. Rietschel, J. Appl. Phys. 85, 7362 (1999); A. K. Tagantsev, V. O. Sherman, K. F. Astafiev, J. Venkatesh, and N. Setter, J. Electroceram. 11, 5 (2003).

${ }^{2}$ J. H. Haeni, P. Irvin, W. Chang, R. Uecker, P. Reiche, Y. L. Li, S. Choudhury, W. Tian, M. E. Hawley, B. Craigo, A. K. Tagantsev, X. Q. Pan, S. K. Streiffer, L. Q. Chen, S. W. Kirchoefer, J. Levy, and D. G. Schlom, Nature (London) 430, 758 (2004).

${ }^{3}$ R. Wördenweber, E. Hollmann, R. Kutzner, and J. Schubert, J. Appl. Phys. 102, 044119 (2007).

${ }^{4}$ W. Chang, J. A. Bellotti, S. W. Kirchoefer, and J. M. Pond, J. Electroceram. 17, 487 (2006).

${ }^{5}$ P. Kužel, F. Kadlec, J. Petzelt, J. Schubert, and G. Panaitov, Appl. Phys. Lett. 91, 232911 (2007).
${ }^{6}$ P. Kužel, C. Kadlec, F. Kadlec, J. Schubert, and G. Panaitov, Appl. Phys. Lett. 93, 052910 (2008).

${ }^{7}$ C. Kadlec, F. Kadlec, H. Němec, P. Kužel, J. Schubert, and G. Panaitov, J. Phys.: Condens. Matter 21, 115902 (2009).

${ }^{8}$ C. Kadlec, V. Skoromets, F. Kadlec, H. Němec, J. Hlinka, J. Schubert, G. Panaitov, and P. Kužel, Phys. Rev. B 80, 174116 (2009).

${ }^{9}$ D. Nuzhnyy, J. Petzelt, S. Kamba, P. Kužel, C. Kadlec, V. Bovtun, M. Kempa, J. Schubert, C. M. Brooks, and D. G. Schlom, Appl. Phys. Lett. 95, 232902 (2009).

${ }^{10}$ R. F. Schaufele, M. J. Weber, and B. D. Silverman, Phys. Lett. 25A, 47 (1967).

${ }^{11}$ J. M. Worlock and P. A. Fleury, Phys. Rev. Lett. 19, 1176 (1967); P. A. Fleury and J. M. Worlock, Phys. Rev. 174, 613 (1968).

${ }^{12}$ I. A. Akimov, A. A. Sirenko, A. M. Clark, J.-H. Hao, and X. X. Xi, Phys. Rev. Lett. 84, 4625 (2000)

${ }^{13}$ M. Born and E. Wolf, Principles of Optics, 7th ed. (Cambridge University Press, New York, 2003).

${ }^{14} \mathrm{~F}$. Gervais, in Infrared and Millimeter Waves, edited by K. J. Button (Academic, New York, 1983), Vol. 8, Chap. 7, p. 279.

${ }^{15}$ W. Zhong, R. D. King-Smith, and D. Vanderbilt, Phys. Rev. Lett. 72, 3618 (1994). 